\title{
Selective Low Concentration Ammonia Sensing in a Microfluidic Lab-on-a-Chip
}

\author{
Bjorn H. Timmer, Koen M. van Delft, W. W. Koelmans, Wouter Olthuis, and Albert van den Berg
}

\begin{abstract}
In the medical community, there is a considerable interest in a diagnostic breath analyzer for ammonia that is selectively enough to measure in exhaled air and small enough for the small volumes available in such an application. An indirect measurement system for low gaseous ammonia concentrations has been miniaturized and integrated on a chip in order to reach this goal. The detection limit of the system was calculated to be 1.1 parts per billion (ppb). The response time was determined to be $1.6 \mathrm{~min}$ with a gas flow of $50 \mathrm{ml} / \mathrm{min}$. The required gas volume for one measurement is therefore sufficiently small, although sampling assistance is required for breath analysis. The selectivity of the system is sufficient to measure ammonia concentrations in the low-ppb range. The system is even sufficiently selective to be used in environments that contain elevated carbon dioxide levels, like exhaled air. The lower ammonia concentration expected in diagnostic breath analysis applications, $50 \mathrm{ppb}$, was demonstrated to be detectable.
\end{abstract}

Index Terms-Gaseous ammonia sensor, microfluidics.

\section{INTRODUCTION}

I $\mathrm{N}$ the medical community, there is a considerable interest in ammonia analyzers that can be applied to measure ammonia levels in exhaled air for the diagnosis of certain diseases [1]. Measuring breath ammonia levels can be a fast diagnostic method for patients with disturbed urea balance, e.g., due to kidney disorder [2] or Heliobacter Pylori bacterial stomach infection [3]-[5]. For such applications, often only a few hundred millileters of exhaled air is available with ammonia concentrations down to $50 \mathrm{ppb}$ in breath of healthy people and up to several hundred pounds per million (ppm) in a diseased state [5] and today no suitable ammonia breath analyzer exists [6]. The ammonia analyzer should be extremely selective because the levels of ammonia are very low compared with other gas concentrations like $\mathrm{O}_{2}$ and $\mathrm{CO}_{2}$ levels.

Many air ammonia detectors have been reported in literature based on different principles [7]-[13]. The most sensitive and selective systems, comprising laser setups, are not suited

Manuscript received February 7. 2005; revised September 19, 2005. This work was supported in part by STW, the Dutch funding agency for university research. The associate editor coordinating the review of this paper and approving it for publication was Prof. Fabien Josse.

B. H. Timmer was with the MESA ${ }^{+}$Research Institute, University of Twente. He is now with the Sensata Technologies Holland b.v., 7602 EM Almelo (e-mail: bios.ewi.utwente.nl).

K. M. van Delft was with the MESA ${ }^{+}$Research Institute, University of Twente, 7500 AE Enschede, The Netherlands. He is now with Philips Semiconductors, Nijmegen, The Netherlands.

W. W. Koelmans, W. Olthuis, and A. van den Berg are with the MESA ${ }^{+}$ Research Institute, University of Twente, 7500 AE Enschede, The Netherlands (e-mail: w.olthuis@ewi.utwente.nl; a.vandenberg@utwente.nl).

Digital Object Identifier 10.1109/JSEN.2006.874020 for miniaturization and integration on a chip and are therefore not applicable for measuring in the small available gas volumes. Methods for direct gas concentration measurement that are more suitable for miniaturization have been shown, like tin oxide film [8] or conducting polymer-film gas sensors [9]. These methods have been applied to measure ammonia concentrations [10]-[12] but most of them showed poor selectivity and inadequate detection limits and are thus not suited.

Other air analyzing systems for measuring ambient gasses make use of gas samplers like denuders or diffusion scrubber [14]-[16]. These systems have the advantage that they can concentrate the ammonia by sampling a volume of gas into a smaller volume of liquid where ammonium ions are formed [17], [18]. Many accurate ways to measure low ammonium concentrations have been shown [19].

An environmental monitoring system for ambient ammonia has been developed at the Energy Research Centre of the Netherlands (ECN) [20] that is accurate enough for breath analysis. However, the apparatus requires an analyte gas flow of 11 per minute for more then $10 \mathrm{~min}$. Miniaturization to reduce the analyte gas consumption is required. It can be expected that this will also make the device more rugged and less sensitive to disturbances [21], [22].

The apparatus comprises a gas sampler, a separator, and a detector [17], [20]. First, gas is sampled into an acid sample solution through a microporous water-repellent membrane. In the selector part, interfering acid gasses are removed and only ammonia will diffuse through a second membrane into a purified water stream. The resulting ammonium concentration is quantified by integrating an electrolyte conductivity (EC) sensor. The system is schematically shown in Fig. 1 .

\section{THEORETICAL}

Normal ambient ammonia concentrations are in the low ppb range; an average of $2 \mathrm{ppb}$ is assumed for the Netherlands [20]. The lower expected ammonia concentration for breath analysis is about $50 \mathrm{ppb}$ [2]. Normal ambient $\mathrm{CO}_{2}$ levels are about 300 $\mathrm{ppm}$. Exhaled air can contain up to a hundredfold more, 30000 $\mathrm{ppm}$. The selectivity of the apparatus should be such that $50 \mathrm{ppb}$ of ammonia is detectable [5].

Ammonia is the main alkaline gas, both in environmental applications and in breath analysis. The used detection principle is based on this fact. First, in the gas sampler, gas is sampled through a microporous hydrophobic membrane into an acid sample solution. In the acid solution, ammonia will react with water and form ammonium ions. This reaction is very fast, and at low $\mathrm{pH}$ values, the equilibrium will cause almost all ammonia to be converted to ammonium [17]. Weak acidifying gasses, like 


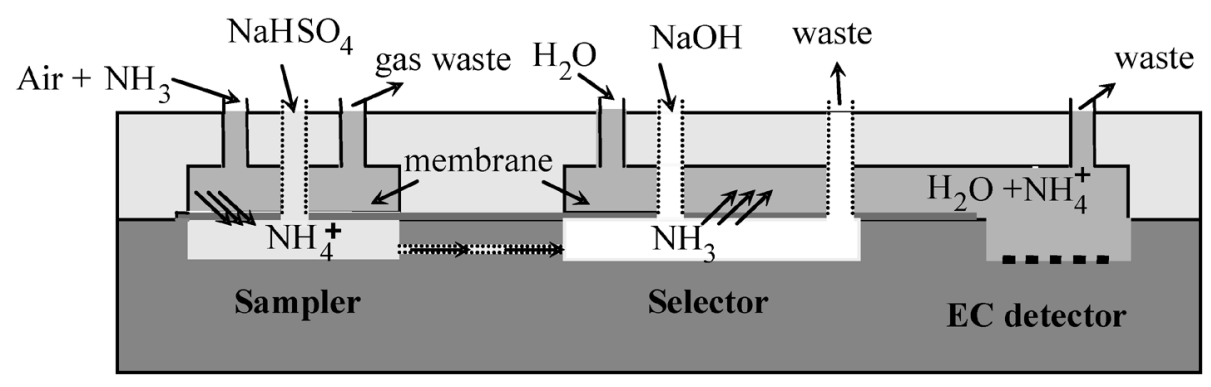

Fig. 1. Schematic of the air ammonia analyzer developed at ECN [19].

carbon dioxide, stay neutral in the acid solution. The overall reaction is given for ammonia and carbon dioxide in (1) (dissociation constants can be found in [17]), as follows:

$$
\begin{aligned}
& \mathrm{NH}_{3}(\mathrm{~g})+\mathrm{H}_{3} \mathrm{O}^{+}(\mathrm{aq}) \stackrel{\mathrm{pH}=3}{\longrightarrow} \mathrm{NH}_{4}^{+}(\mathrm{aq}) \\
& \mathrm{CO}_{2}(\mathrm{~g})+\mathrm{H}_{2} \mathrm{O}(\mathrm{l}) \stackrel{\mathrm{PH}=3}{\longrightarrow} \mathrm{CO}_{2}(\mathrm{aq})+\mathrm{H}_{2} \mathrm{O}(\mathrm{l}) \leftrightarrow \mathrm{H}_{2} \mathrm{CO}_{3}(\mathrm{aq}) .
\end{aligned}
$$

Subsequently, the sample solution is pumped into the selector where the $\mathrm{pH}$ is increased by adding a strong alkaline solution. The high $\mathrm{pH}$ value shifts the equilibrium, neutralizing the ammonium ions back to ammonia and dissociating sampled acid gasses like $\mathrm{CO}_{2}$, as described by (2) [17]

$$
\begin{aligned}
& \mathrm{NH}_{4}^{+}(\mathrm{aq})+\mathrm{OH}^{-}(\mathrm{aq}) \stackrel{\mathrm{pH}=11}{\longrightarrow} \mathrm{NH}_{3}(\mathrm{aq}) \leftrightarrow \mathrm{NH}_{3}(\mathrm{~g})+\mathrm{H}_{2} \mathrm{O}(\mathrm{l}) \\
& \mathrm{H}_{2} \mathrm{CO}_{3}(\mathrm{aq})+\mathrm{OH}^{-}(\mathrm{aq}) \stackrel{\mathrm{pH}=11}{\longrightarrow} \mathrm{CO}_{3}^{2-}(\mathrm{aq})+2 \mathrm{H}_{2} \mathrm{O}(\mathrm{l}) .
\end{aligned}
$$

The selector is formed by two opposite channels separated by a gas permeable, water-repellent polypropylene membrane, as illustrated in Fig. 1. The opposite channel is flushed with purified water. The dissolved ammonia turns gaseous at the phase separating membrane interface and can pass through the membrane into the purified water where it partly dissolves. The dissolved ammonia reacts with water due to the dissociation equilibrium, as follows:

$$
\mathrm{NH}_{3}(\mathrm{~g})+\mathrm{H}_{2} \mathrm{O}(\mathrm{l}) \leftrightarrow \mathrm{NH}_{4}^{+}(\mathrm{aq})+\mathrm{OH}^{-}(\mathrm{aq}) .
$$

All remaining ionized acid gasses are flushed out of the system as waste. The formed ammonium ions in the water stream are quantified using an electrolyte conductivity detector [24].

The obtainable selectivity is a function of the described chemical reactions. The Henry constant relates the concentrations in the gas sampler of the gaseous and the dissolved state. The concentration levels of the ions that are formed due to the dissociation reactions can be calculated using the dissociation constants of the equilibriums. Furthermore, the $\mathrm{pH}$ values of the solutions in the gas sampler, selector, and the detector stream should be known. These values, however, change when gasses like $\mathrm{CO}_{2}$ and $\mathrm{NH}_{3}$ are sampled and are therefore influenced by the amount of sampled gasses, the used internal volumes, and liquid flows as well as the speed at which the involved reactions occur. In a previous publication, a detailed analysis was made that indicates that the desired selectivity can be reached, even for breath analysis conditions [17]. For breath analysis, the system described in this paper with an analyte gas to sample solution ratio of 3125 and an initial $\mathrm{pH}$ of 3.5 would sample more than $99.9 \%$ of the ammonia that is ionized for more than $99.9 \%$, where only $0.001 \%$ of the $\mathrm{CO}_{2}$ is sampled. After addition of the alkaline solution in the selector part of the system, $99.9 \%$ of the formed ammonium ions are transformed into gaseous ammonia again where only $3.5 \cdot 10^{-4 \%}$ of the dissolved $\mathrm{CO}_{2}$ is available in the gaseous state. Based on this estimation, a selectivity toward ammonia compared with $\mathrm{CO}_{2}$ of several millions is expected.

\section{EXPERIMENTAL}

\section{A. Miniaturized Detection System}

The system shown schematically in Fig. 1 has been realized as an integrated miniaturized detection system. The key components, the gas sampler, the selector, and the electrolyte conductivity sensor have been realized in one chip. Both the gas sampler and the selector comprise two opposite channels separated by a gas-permeable water-repellent membrane. A membrane suitable for integration in a microfluidic gas detection system is microporous polypropylene (PP). These membranes are both gas permeable and water repellent, and they can be glued to glass and silicon using epoxy glue. The thickness of commercial microporous PP membranes, acquired from Schleider \& Schuell (PP $0.22 \mu \mathrm{m}$ hydrophobic membrane), is about $150 \mu \mathrm{m}$. Previous research taught us that the channels of the system are required to have a depth of at least $15 \mu \mathrm{m}$ and a maximum width of about $1 \mathrm{~mm}$ to prevent clogging of the channel by buckling of the membrane into the channel.

The concentration enhancement ratio and thus the selectivity can be optimized by making the gas sampler as large as possible to be able to pump through a large gas flow and the selector as small as possible to reduce the flow through time of the selector solution. The depth of the sample solution and the selector solution channel was chosen as shallow as possible, $15 \mu \mathrm{m}$, to reduce diffusion distances. The gas channel of the sampler could be made deeper since gaseous diffusion is much faster. The optimized dimensions of the system have been obtained from mass transport simulations conducted in the finite-element program CFD-ACE from CFD Research Corporation.

Shallow channel structures, all channels except the gas channel have a depth of $15 \mu \mathrm{m}$ and have been etched in glass using wet chemical etching. Borofloat glass wafers were first 


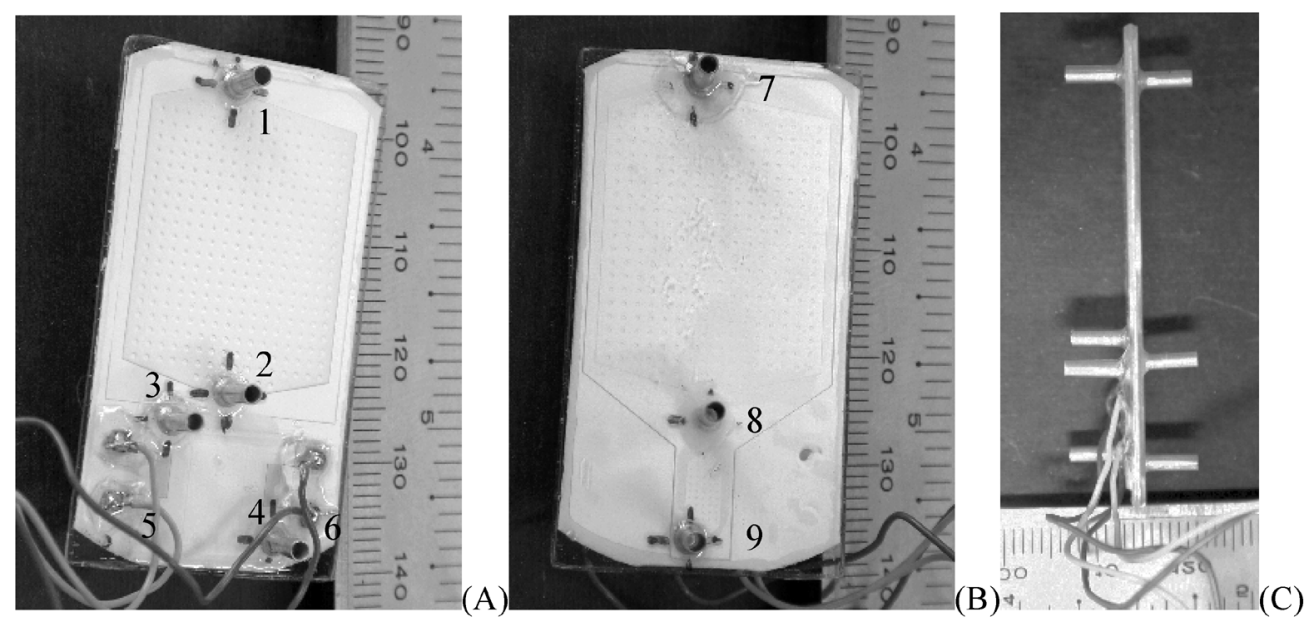

Fig. 2. Photographs of an assembled miniaturized ammonia analyzer showing (a) a top view, (b) a bottom view, and (c) a side view of the chip that comprises of a microporous membrane glued between two micromachined glass layers with 1-gas outlet, 2-gas inlet, 3-purified water inlet, 4-water outlet, 5-EC detector at the water inlet, 6-EC detector at the water outlet, 7—sample solution inlet, 8-alkaline selector solution inlet, and 9—solution outlet.

cleaned in fuming nitric acid. A chromium mask layer was deposited and structured using standard photolithography and wet chromium etching. Subsequently $15 \mu \mathrm{m}$ deep channels were etched in the glass in a $10 \%$ HF solution. The used photoresist was stripped from the wafer in acetone. Through-holes and deep channels like the gas channel with a depth of $100 \mu \mathrm{m}$ were made using powder blasting [25]. A photosensitive protective foil, Ordyl BF410, was laminated onto the wafer and structured using photolithography and developed in sodium carbonate. After powder blasting the through-holes with $9 \mu \mathrm{m}$ aluminum oxide powder, the foil was stripped in sodium carbonate and the wafer was ultrasonically cleaned in acetone to remove powder residue and diced.

A miniaturized EC detector optimized for measuring low ion concentrations in small volumes has been realized in previous research [24]. A comb-structured two electrodes conductivity detector with 95 electrode fingers with a width of $10 \mu \mathrm{m}$, a length of $1270 \mu \mathrm{m}$ and a spacing between the fingers of $30 \mu \mathrm{m}$ has been realized, resulting in a cell constant of $15 \mathrm{~m}^{-1}$. After cleaning a Pyrex glass wafer, a negative image of the designed electrode structure was created on the wafer in photoresist using standard photolithography. After sputtering a chromium adhesion layer, a platinum electrode layer was sputtered over the entire wafer. The electrode structure was realized using a liftoff step by ultrasonically removing the photoresist layer. A glass cover to seal the electrodes was made by etching channels and powder blasting through holes. The cover was glued over the electrodes using epoxy glue. Two detectors were integrated in the system, one at the water inlet and one at the water outlet. The inlet detector is used as an indicator for changes that are not caused by a change in analyte gas, like temperature changes or pollutants in the purified water source.

The different parts were assembled by gluing the two glass parts together with the membrane in between. Fluid and gas connectors as well as wires were connected to the inlet and outlet holes and the bond pads of the electrodes, respectively. A photo of the integrated ammonia measurement system is shown in Fig. 2.

\section{B. Measurement Setup}

A $50 \mathrm{ml} / \mathrm{min}$ analyte gas flow was applied to the gas sampler. Pure nitrogen, pure $\mathrm{CO}_{2}$, and a mixture of nitrogen with $10 \mathrm{ppm}$ $( \pm 10 \%)$ ammonia were acquired from Hoekloos. Gas flow was controlled using mass flow controllers from Bronkhorst Hightech, EL-flow F-110C. The mass flow controllers used for the ammonia and the $\mathrm{CO}_{2}$ source have a maximum flow of $3 \mathrm{ml} / \mathrm{min}$ with a minimum controllable flow of $2 \%$, which is $60 \mu \mathrm{l} / \mathrm{min}$. The nitrogen flow was controlled with a $100 \mathrm{ml} / \mathrm{min}$ mass-flow controller. The mass-flow controllers should always be open, at least at $2 \%$, to prevent back flow of the analyte gas, resulting in minimum attainable ammonia and $\mathrm{CO}_{2}$ concentrations of 12 ppb and $1000 \mathrm{ppm}(0.1 \% \mathrm{v})$ respectively, at a $50 \mathrm{ml} / \mathrm{min}$ gas flow.

The three liquid flows, the sample solution, selector solution, and purified water flow, were pressure driven using a CMA 102 microdialysis syringe pumps connected to a personal computer. Deionized water was purified by an ion exchange column filled with a Baker mixed-bed ion exchange resin.

The conductance between the electrodes in the detector was measured with homemade interface electronics that were connected to the same computer. The measurement frequency of the interface electronics was optimized to reduce capacitive influences to $1 \mathrm{kHz}$ at a $66 \mathrm{mV}_{\text {rms }}$ sinusoidal measurement signal [24]. A measurement and control Labview program was used to be able to adjust flow settings and perform measurements at the same time. A schematic of the used measurement setup is shown in Fig. 3.

\section{Measurement Protocol}

A $325 \mu \mathrm{M} \mathrm{NaHSO}_{4}$ sample solution, with a $\mathrm{pH}$ of 3.5, was pumped through the gas sampler at $16 \mu \mathrm{l} / \mathrm{min}$. A $0.1 \mathrm{M} \mathrm{KOH}$ solution was added to the selector, with a $\mathrm{pH}$ of 13.0, at a flow rate of $4 \mu \mathrm{l} / \mathrm{min}$. The purified water was pumped into the selector at a flow rate of $2 \mu \mathrm{l} / \mathrm{min}$. Prior to the experiments, a calibration measurement was performed with the electrolyte conductivity 


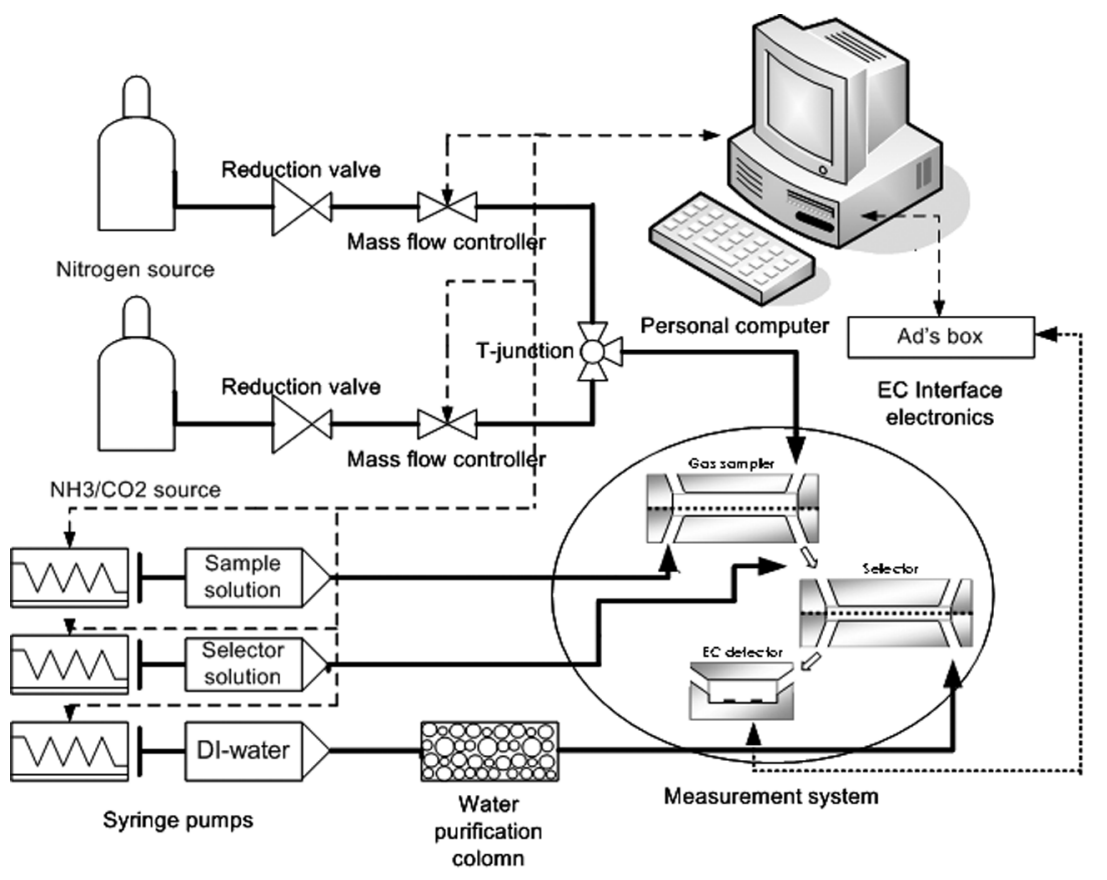

Fig. 3. Schematic representation of the used measurement setup.

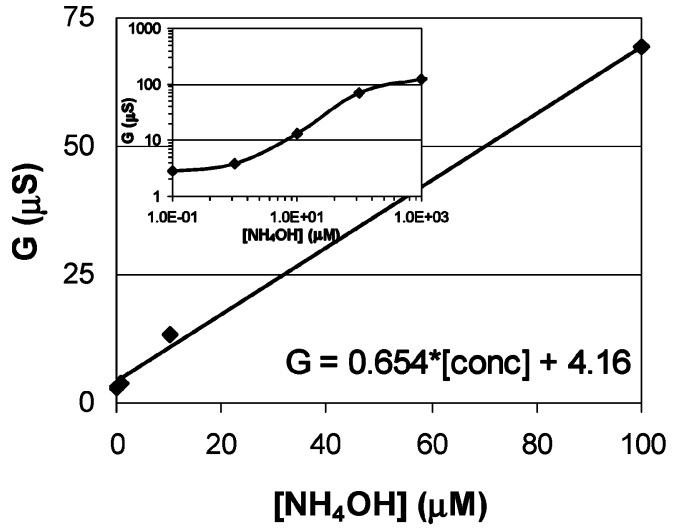

(A)

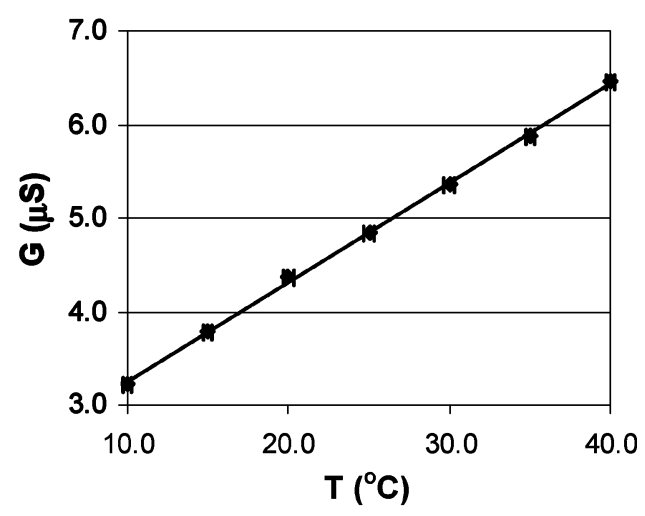

(B)

Fig. 4. EC detector calibration with $\mathrm{NH}_{4} \mathrm{OH}$ solutions at (a) room temperature and (b) temperature dependence of the purified water conductance between $10{ }^{\circ} \mathrm{C}$ and $40{ }^{\circ} \mathrm{C}$.

detectors. A solution with a known ammonium hydroxide concentration was flushed through the detector.

\section{RESULTS AND DISCUSSION}

The calibration result of the detector is shown in Fig. 4(a). For the higher concentration range, the used measurement frequency was relatively low. This can be noticed from the flattening of the curve at concentrations above $100 \mu \mathrm{M}$, as shown in the inset. The lower conductance at these higher electrolyte concentrations causes an increased influence of the double layer capacitance at the electrode-electrolyte interface. The interface electronics do not measure at the optimal frequency anymore. For lower ion concentrations, up to 100 $\mu \mathrm{M}$, the relation between the concentration and the measured conductance was linear within $2 \%$. The equation of the linear fit was used in the rest of this paper as the relation to calculate the conductance as a function of the $\mathrm{NH} 4 \mathrm{OH}$ concentration.
It should be noted that this calibration is only valid at room temperature, $20^{\circ} \mathrm{C}$, since electrolyte conductivity is also a function of the temperature. The temperature dependence of the conductance measured with purified water is plotted in Fig. 4(b), showing a temperature dependence of $2.4 \pm 0.3 \% /{ }^{\circ} \mathrm{C}$ with respect to the conductance at $20^{\circ} \mathrm{C}$.

The first experiment conducted with the measurement system was an ammonia sensitivity test. The system was first flushed with pure nitrogen for an hour. Once the output signal of the detectors became stable after cleaning of the selector channels and detector electrodes with purified water, ammonia was added to the nitrogen carrier gas. The measurement result obtained with an increasing ammonia concentration is shown in Fig. 5.

It is shown that the sensor responds to an increase in ammonia concentration. The response time of the device seems to be a function of the ammonia concentration. This is an artefact caused by the analyte gas preparation setup shown in Fig. 4 . 


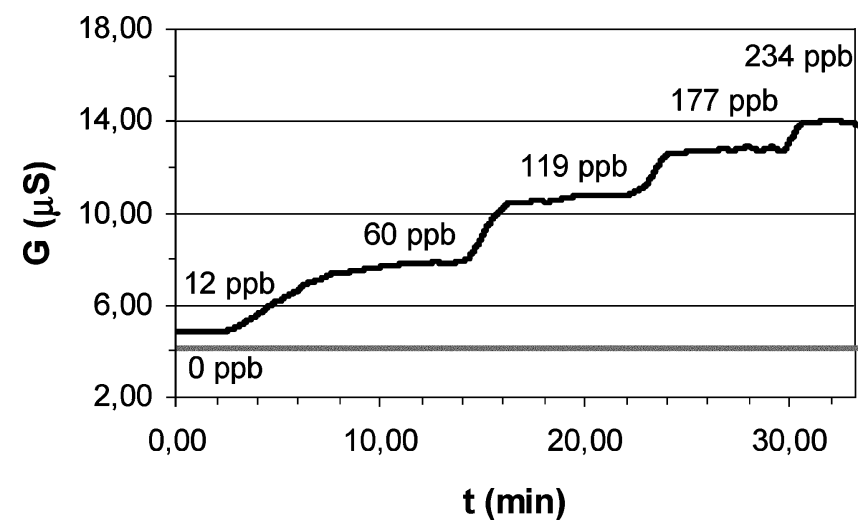

Fig. 5. Ammonia sensitivity experiment showing the measured conductance of the two detectors at the water inlet and the water outlet as a function of the ammonia concentration in the analyte gas.

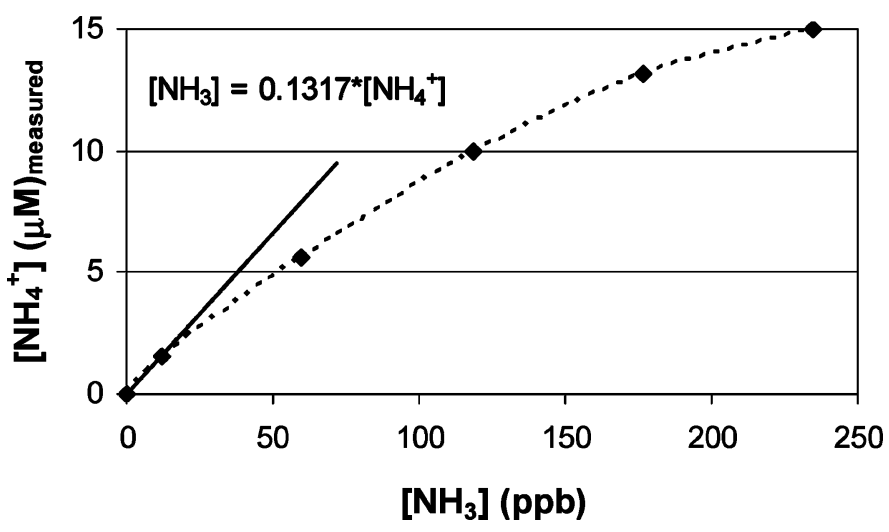

Fig. 6. Conductance as a function of the applied ammonia concentration with a linear fit through the lowest concentrations to determine the slope of the relation.

Teflon tubing was used as interconnect to transport the gasses from the mass-flow controllers to a T-junction that was connected with the gas inlet of the measurement system. When making very low ammonia concentrations, the flow from the source is so slow that it takes more than a minute before the desired gas composition actually reaches the sensor. Diffusion inside the tubing causes dispersion of the step in concentration. The response time, the time it takes for the signal to reach $90 \%$ of the end value, was thus several minutes. When the flow from the ammonia source was higher, the response time of the measurement system itself becomes the dominant factor, resulting in a response time of $1.6 \mathrm{~min}$. Although this is a significant reduction compared with the large-scale apparatus that this device was based on, an additional breath sampling device will still be required.

In order to determine the detection limit of the system, the ammonium concentration corresponding to the measured conductance values is plotted in Fig. 6 as a function of the applied ammonia concentration. The ammonium concentration was calculated using the concentration-conductance relation determined from the calibration measurement of which the result was shown in Fig. 4(a). The error bars indicate three times the standard deviation of the conductance during the last minute before the concentration was increased, taken from Fig. 4.

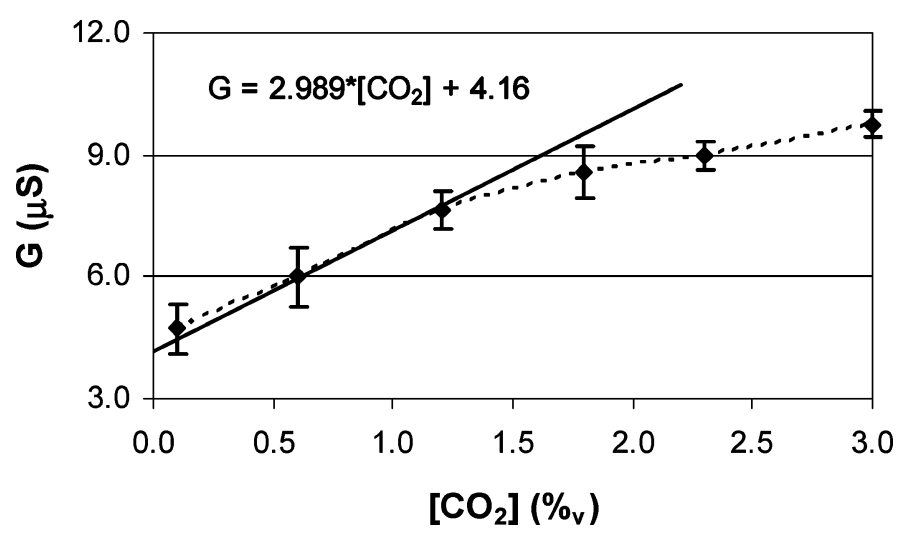

Fig. 7. Conductance measurement result with $\mathrm{CO}_{2}$.

Manually, a fit to the curve was made through the lowest applied concentration of $12 \mathrm{ppb}$ and the response to ammonia-free nitrogen. Three times the standard deviation value of the response to $12 \mathrm{ppb}$, the noise level of the first "flat" area in the measurement shown in Fig. 6, was calculated to be $0.14 \mu \mathrm{M}$. With this value and the relation, the theoretical detection limit was calculated to be $1.1 \mathrm{ppb}-$ a significant improvement compared with previous results obtained with a modular sensor, described in [17], where a detection limit of several hundred ppb was obtained. This lower detection limit could be further lowered by reducing the deviation in output signal, which seems to be caused mainly by flow variations inside the system. Gas flow variations in particular largely influence the conductance signal.

The above results showed that the system was fast and accurate enough for a diagnostic breath analysis tool. For such a system, however, a high selectivity toward ammonia is required. In exhaled-breath carbon dioxide levels are much higher than in normal air, as discussed in the introduction. To investigate the selectivity of the measurement system, the conductance response to a $50-\mathrm{ml} / \mathrm{min}$ analyte gas of nitrogen containing known partial pressures of $\mathrm{CO}_{2}$ concentrations was determined. The result is shown in Fig. 7.

In Fig. 7, it is shown that the measurement system did respond to increasing partial $\mathrm{CO}_{2}$ pressures in the analyte gas. The maximum tested $\mathrm{CO}_{2}$ concentration, 3 vol., corresponds to the higher level that can be found in exhaled breath. The resulting conductance value can be compared with the ammonia measurement result shown in Fig. 5 and corresponds to an ammonia concentration of about $90 \mathrm{ppb}$. This means the selectivity of the system toward 3 vol. \% of $\mathrm{CO}_{2}$ and ammonia is about 330000 . It was our goal to reach a selectivity of 600000 so that the conductance response to the $\mathrm{CO}_{2}$ level in exhaled breath would be comparable with the desired ammonia detection limit of $50 \mathrm{ppb}$. Although this selectively was not reached completely, exhaled $\mathrm{CO}_{2}$ levels are relatively stable [23]. Therefore, it still seems possible to detect $50 \mathrm{ppb}$ of ammonia. Further tests are required with both $\mathrm{CO}_{2}$ and $\mathrm{NH}_{3}$, or even better exhaled air, which could unfortunately not be conducted with the used measurement setup.

With the linear fit to the lower carbon dioxide concentration responses forced through the conductance level at pure nitrogen, as shown in Fig. 7, an estimation of the system response to 
normal atmospheric $\mathrm{CO}_{2}$ concentrations can be made. A concentration of $300 \mathrm{ppm}$ or 0.03 vol. \% corresponds to a conductance of $4.24 \mu \mathrm{S}$. This equals the response of the system to an ammonia concentration of $1.0 \mathrm{ppb}$, according to the results shown in Figs. 4 and 6. This means that the system can be used for environmental monitoring in areas where the ammonia concentration is in the low-ppb range.

\section{CONCLUSION}

A miniaturized measurement system for low gaseous ammonia concentrations has been realized on a chip. The system comprises three main parts: a gas sampler that converts gaseous ammonia to dissolved ammonium ions, a selector that removes interfering gasses like $\mathrm{CO}_{2}$, and a detector that quantifies the electrolyte concentration that is directly proportional to the ammonia concentration in the analyte gas. A phase separation membrane was integrated by gluing polypropylene membranes between two glass chips comprising micromachined channels.

The detection limit of the system was calculated to be $1.1 \mathrm{ppb}$. The response time of the measurement system was determined to be $1.6 \mathrm{~min}$. In environments with normal acidifying gas concentrations, the selectivity of the system is sufficient to measure ammonia concentrations in the low-ppb range. The system is even sufficiently selective to ammonia to be used in environments that contain elevated carbon dioxide levels, like exhaled air. The lower ammonia concentration expected in diagnostic breath analysis applications, $50 \mathrm{ppb}$, is easily detectable. It should be noted, however, that a breath sampling device is required because 1.6-min sampling time is too long for directly measure exhaled breath.

The system comprises two electrolyte conductivity detectors: one to determine the conductance of the water that is pumped into the system and one to quantify the ammonium concentration as a result of the ammonia uptake. This first detector can be used as an indicator for sudden changes in input boundary conditions like pollution in the water flow or changes in temperature. It is shown that the temperature dependency of the water conductance is nearly linear at temperatures in the range from $10{ }^{\circ} \mathrm{C}$ to $40{ }^{\circ} \mathrm{C}$.

\section{ACKNOWLEDGMENT}

The authors gratefully acknowledge the technical support from D. Hermes on the realization of the chips and A. Sprenkels for developing the interface electronics.

\section{REFERENCES}

[1] W. Ament, J. R. Huizenga, E. Kort, T. W. van der Mark, R. G. Grevink, and G. J. Verkerke, "Respiratory ammonia output and blood ammonia concentration during incremental exercise," Int. J. Sports Med., vol. 20, pp. 71-77, 1999.

[2] L. R. Narasimhan, W. Goodman, C. Kumar, and N. Patel, "Correlation of breath ammonia with blood urea nitrogen and creatinine during hemodialysis," Proc. Nat. Acad. Sci., vol. 98, no. 8, pp. 4617-4621, 2001.

[3] B. Marshall and J. R. Warren, "Unidentified curved bacillus and gastric Epithaeium in active chonic gastritis," Lancet, vol. 1, pp. 1273-1275, 1993.

[4] J. C. E. Underwood, General and Systematic Pathology, 2nd ed. London, U.K.: Churchill Livingstone, 1996, pp. 414-415.

[5] D. J. Kearney, T. Hubbard, and D. Putnam, "Breath ammonia measurement in Helicobacter pylori infection," Dig. Diseases Sci., vol. 47, no. 11 , pp. 2523-2530, 2002.
[6] E. Verpoorte, "Microfluidic chips for clinical and forensic analysis," Electrophoresis, vol. 23, pp. 677-712, 2002.

[7] S. G. Buckly, C. J. Damm, W. M. Vitovec, L. A. Sgro, R. F. Sawyer, C. P. Koshland, and D. Lucas, "Ammonia detection and monitoring with photofragmentation fluorescence," Appl. Opt., vol. 37, no. 36, pp. 8382-8391, 1998.

[8] R. K. Sharma, P. C. H. Chan, Z. Tang, G. Yan, I.-M. Hsing, and J. K. O. Sin, "Investigation of stability and reliability of tin oxide thin-film for integrated micro-machined gas sensor devices," Sens. Actuators B, Chem., vol. 81, pp. 9-16, 2001.

[9] F. Zee and J. W. Judy, "Micromachined polymer-based chemical gas sensor array," Sens. Actuators B, Chem., vol. 72, pp. 120-128, 2001.

[10] M. Aslam, V. A. Chaudhary, I. S. Mulla, S. R. Sainkar, A. B. Mandale, A. A. Belhekar, and K. Vijayamohanan, "A highly selective ammonia gas sensor using surface-ruthenated zinc oxide," Sens. Actuators A, Phys., vol. 75, pp. 162-167, 1999.

[11] I. Laehdesmaeki, A. Lewenstam, and A. Ivaska, "A polypyrrole-based amperometric ammonia sensor," Talanta, vol. 43, pp. 125-134, 1996.

[12] V. V. Chabukswar, S. Pethkar, and A. A. Athawale, "Acrylic acid doped polyaniline as an ammonia sensor," Sens. Actuators B, Chem., vol. 77, pp. 657-663, 2001.

[13] J. Maier, "Electrochemical sensor principles for redox-active and acidbase-active gases," Sens. Actuators B, Chem., vol. 65, pp. 199-203, 2000.

[14] P. K. Simon, P. K. Dasgupta, and Z. Vecera, "Wet effluent denuder coupled liquid/ion chromatography systems," Anal. Chem., vol. 63, pp. 1237-1242, 1991.

[15] P. F. Lindgren and P. K. Dasgupta, "Measurement of atmospheric sulfur dioxide by diffusion scrubber coupled ion chromatography," Anal. Chem., vol. 61, pp. 19-24, 1989.

[16] C. B. Boring, R. Al-Horr, Z. Genfa, and P. K. Dasgupta, "Field measurement of acid gases and soluble anions in atmospheric particulate matter using a parallel plate wet denuder and an alternating filter based automatic analysis system," Anal. Chem., vol. 74, pp. 1256-1268, 2002.

[17] B. H. Timmer, K. M. van Delft, R. P. Otjes, W. Olthuis, and A. v. d. Berg, "A miniaturized measurement system for ammonia in air," Analytica Chimica Acta, vol. 507, no. 1, pp. 139-145, 2004.

[18] B. H. Timmer, W. Olthuis, and A. v. d. Berg, "Sampling small volumes of ambient ammonia using a miniaturized gas sampler," Lab on a Chip, no. 4, pp. 252-255, 2004.

[19] G. Schultze, C. Y. Liu, M. Brodowski, and O. Elsholz, "Different approaches to the determination of ammonium ions at low levels by flow injection analysis," Analytica Chimica Acta, vol. 214, pp. 121-136, 1988.

[20] J. W. Erisman, R. Otjes, A. Hensen, P. Jongejan, P. v. d. Bulk, A. Khlystov, H. Möls, and S. Slanina, "Instrument development and application in studies and monitoring of ambient ammonia," Atmos. Environ., vol. 35, pp. 1913-1922, 2001.

[21] A. van den Berg and T. S. J. Lammerink, "Micro total analysis systems: Microfluidic aspects, integration concept and applications," Top. Current Chem., vol. 194, pp. 21-50, 1997.

[22] A. Manz, N. Graber, and H. M. Widmer, "Miniaturized total chemical analysis systems-A novel concept for chemical sensing," Sens. Actuators B, Chem., vol. 1, pp. 244-248, 1990.

[23] R. M. Berne and M. N. Levy, Physiology, 4th ed. St. Louis, MO: Mosby, 1998.

[24] B. H. Timmer, W. Sparreboom, W. Olthuis, P. Bergveld, and A. v. d. Berg, "Optimization of an electrolyte conductivity detector for measuring low ion concentrations," Lab on Chip, vol. 2, pp. 121-124, 2002.

[25] S. Schlautmann, H. Wensink, R. B. M. Schasfoort, M. Elwenspoek, and A. v. d. Berg, "Powder blasting technology as an alternative tool for microfabrication of capillary electrophoresis chips with integrated conductivity electrodes," J. Micromech. Microeng., vol. 11, no. 4, pp. 386-389, 2001.

Bjorn H. Timmer was born in 1975. He received the M.Sc. degree in electrical engineering from the University of Twente, Enschede, The Netherlands, in 2000 , focusing on a microdosing system for calibrating a miniaturized bedside monitoring system and the Ph.D. degree from the Laboratory of Biosensors, Lab-on-a-Chip group, part of the MESA+ Institute for Nanotechnology, University of Twente, in 2004, where his research focused on the miniaturization and optimization of a micro-fluidic ammonia sensor for measuring low-ppb levels of ambient ammonia, in cooperation with the Energy Research Centre of the Netherlands $(\mathrm{ECN})$.

Since 2004, he has been working at Sensata Technologies Holland b.v., a former division of Texas Instruments, as a Sensor Products Design Engineer. 
Koen M. van Delft was born in 1978. He received the Bachelor's degree in precision engineering, with a specialization in microsystem technology, from the Hogeschool Utrecht, The Netherlands, in 2001 and the M.Sc. degree in applied physics from the University of Twente, Enschede, The Netherlands, in 2006.

From 2001 to 2006, he was working as a Research Technician in the Lab-on-a-Chip group, part of the MESA+ Institute for Nanotechnology, University of Twente. Currently, he is working as a Reliability Engineer on qualification and reliability strategies within Philips Semiconductors, Nijmegen, The Netherlands.

W. W. Koelmans was born in 1981. He received the Bachelor's degree from the University of Twente, Enschede, The Netherlands, where his assignment was with the Lab-on-a-Chip group. He is currently working towards the Master's degree in electrical engineering at the group of Systems and Materials for Information Storage of the same university, where his research focuses on fabricating and coating tips for spin polarized scanning tunneling microscopy.

Wouter Olthuis was born in 1960. He received the M.Sc. degree in electrical engineering from the University of Twente, Enschede, The Netherlands, in 1986 on the subject of thermally excited resonating silicon membrane pressure sensors and the Ph.D. degree from the Faculty of Electrical Engineering, University of Twente, in 1990 with a dissertation focusing on the use of iridium oxide in ISFET-based coulometric sensor-actuator devices.

In 1986, he joined the Center for MicroElectronics, Enschede (CME), doing research on inorganic electret materials for subminiature silicon microphones.
Since 1991, he has been an Assistant Professor in the Laboratory of Biosensors, the Lab-on-a-Chip group, part of the MESA ${ }^{+}$Research Institute, University of Twente, and as such cosupervising many projects on both physical and (bio)chemical sensors and sensor systems for medical and environmental applications.

Albert van den Berg received the Master's degree in applied physics from the University of Twente, Enschede, The Netherlands, in 1983, with a thesis focusing on on the topic of chemically modified ISFETs.

From 1988 to 1990, he was Project Manager in the Chemical Sensor Department at the Swiss Center for Microelectronics and Microtechnology (CSEM), Neuchâtel, Switzerland. From 1990 to 1993, he did research on miniaturized chemical sensors and sensor systems at the IMT, University of Neuchâtel, Switzerland. From 1993 until 1999, he was Research Coordinator for Micro Total Analysis Systems ( $\mu \mathrm{TAS})$ at $\mathrm{MESA}^{+}$, University of Twente, later extended to Miniaturized Chemical Systems (MiCS). In 1998, he was appointed as Full Professor in the Lab-on-a-Chip group, University of Twente. He has published over 100 peer-reviewed papers and edited several books on Labs-on-a-Chip. His current research interests focus on analysis systems and sensors, microreactors, microfluidics, and nanofluidics, and Lab-in-a-Cell.

Mr. van den Berg is and has been member of numerous scientific committees ( $\mu$ TAS, MEMS, Micromachine Summit, Transducers, Nanotech) and served as editor of the section $\mu$ TAS of Sensors and Actuators B. In 2002, he received the "Simon Stevin Master" Award from the Dutch Technical Science Foundation. 